\title{
Comparación económica entre el sistema tradicional y el sistema intensivo de la producción de arroz en el Ecuador
}

\section{Economic comparison between the traditional system and the intensive system of rice production in Ecuador}

DOI: $10.46932 / \mathrm{sfjdv3n1-076}$

Received in: Jan 30st, 2021

Accepted in: Feb 1th, 2022

\author{
Jorge Washington Cedeño Plaza \\ Estudiante carrera de economía agropecuaria \\ Universidad Técnica de Machala Km 5 1⁄2 vía Pasaje \\ Machala-Ecuador \\ E-mail: jcedeno3@utmachala.edu.ec \\ Jessica Maribel Quezada Campoverde \\ Ingeniera agrónoma \\ Universidad Técnica de Machala Km 5 1⁄2 vía Pasaje \\ Machala-Ecuador \\ E-mail:jquezada@utmachala.edu.ec \\ Víctor Javier Garzón Montealegre \\ Economista \\ Universidad Técnica de Machala Km 5 1⁄2 vía Pasaje \\ Machala-Ecuador \\ E-mail: vgarzon@utmachala.edu.ec \\ Salomón Alejandro Barrezueta Unda \\ Ingeniero Agrónomo. PhD. \\ Universidad Técnica de Machala Km 5 1⁄2 vía Pasaje \\ Machala-Ecuador \\ E-mail: sabarrezueta@utmachala.edu.ec \\ Marcos Antonio Espinosa Aguilar \\ Economista \\ Universidad Técnica de Machala Km 5 1⁄2 vía Pasaje \\ Machala-Ecuador \\ E-mail: maespinos@utmachala.edu.ec
}

\section{RESUMEN}

Mediante la comparación económica entre el Sistema Tradicional y el Sistema Intensivo de la Producción de Arroz, se estima que el sistema tradicional requiere una mayor inversión de capital por hectárea de producción, lo cual generan que sus ganancias sean menores. El Sistema Intensivo de la Producción Arroz genera un mayor crecimiento de la planta, mayor resistencia a los cambios climáticos y a las plagas, al tener una distancia adecuada de siembra. Son pocos los países en Sudamérica que confían en el Sistema Intensivo de la Producción de Arroz, el mismo que hasta el momento ha generado conformidad en los pequeños productores que ven dentro de este sistema una oportunidad de mayor rentabilidad en sus 
cultivos de arroz. Dentro del objetivo de investigación es comparar los resultados económicos entre el Sistema Tradicional y el Sistema Intensivo de la Producción de Arroz en Ecuador. Saber ¿Cuál es el beneficio de la implementación del Sistema Intensivo de la Producción de Arroz en Ecuador? Los resultados recomiendan que este nuevo sistema de producción genera un incremento favorable dentro del cultivo, en lo económica, reduce el costo de producción y son mayores los beneficios obtenidos de este nuevo sistema al contrario del tradicional.

Palabras claves: Gramínea, Rendimiento, Pequeños Agricultores, Oryza Sativa.

\begin{abstract}
Through the economic comparison between the Traditional System and the Intensive Rice Production System, it is estimated that the traditional system requires a higher capital investment per hectare of production, which generates lower profits. The Intensive Rice Production System generates greater plant growth, greater resistance to climate change and pests, by having an adequate planting distance. Few countries in South America trust in the Intensive System of Rice Production, the same one that until now has generated conformity in small producers who see within this system an opportunity for greater profitability in their rice crops. Within the research objective is to compare the economic results between the Traditional System and the Intensive System of Rice Production in Ecuador. Know What is the benefit of the implementation of the Intensive Rice Production System in Ecuador? The results recommend that this new production system generates a favorable increase within the crop, economically, it reduces the cost of production and the benefits obtained from this new system are greater than the traditional one.
\end{abstract}

Keywords: Grass, Performance, Small Farmers, Oryza Sativa.

\title{
1 INTRODUCCIÓN
}

El cultivo de arroz (Oryza Sativa), es la especie más predominante de la familia de las gramíneas y su fruto es plenamente consumible, sus orígenes son del Sureste Asiático y su cultivo tiene 10000 años, los hallazgos arqueológicos en China indican que el arroz podría darse entre 2750 y el 3280 a.C, Tailandia 3500 a.C, luego su expansión se dio hacia Camboya y Vietnam, fueron desde estos lugares donde se trasladó sus diferentes especies (Bernis \& Pàmies, 2004).

El arroz es uno de los cereales más cultivados a nivel mundial por representar el alimento básico de más de la mitad de la población mundial, alrededor de 100 millones de hogares de Asia y África dependen de esta actividad como fuente principal de ingresos económicos a sus hogares. La mayor parte de la producción de arroz a nivel mundial es dada por pequeños agricultores y su consumo es casi en su totalidad local (Chang, 2008).

El Ecuador es un país productor y consumidor de arroz el $83 \%$ del cultivo se localiza en el Guayas y los Ríos, la producción de arroz se concentra en la región Costa con un 98.8\% de la producción nacional y en la Sierra $0.5 \%$ y la Amazonia $0.7 \%$ del total nacional. Cada año Ecuador tiene complicaciones dentro de los cultivos de arroz por las diferentes sequias e inundaciones que golpean a los cultivos de arroz (Quijije, Carvajal, Garcia, \& Cedeño, 2019). 


\subsection{EL ARROZ EN AMÉRICA}

En Latinoamérica el cultivo de arroz es en gran parte es un sistema tradicional, dejando en mínimo la implementación de técnicas novedosas coma el sistema intensivo de la producción de arroz, el mismo que busca la maximización de la producción con menos recursos, estimulando a la producción con menos semilla y menor espacio de competición entre plantas, generando un mayor rendimiento.

Gran parte de los cultivos arroceros provienen de pequeños agricultores de países de bajos ingresos y en vías de desarrollo. A consecuencia de falta de recursos y lo complicado de esta producción es importante la implementación de nuevas técnicas las mismas que buscan una mayor calidad del producto, tratando de reducir los costos para que de esta manera se pueda obtener mayor rentabilidad (Shiguango, 2014).

\subsection{PAÍSES QUE MAYOR PRODUCEN ARROZ EN SUDAMÉRICA}

El arroz en Latinoamérica es el cuarto producto mayor consumido, representa el $11 \%$ de las ingestas calóricas per capital de los países, lo que representa una base fundamental dentro de la seguridad alimenticia. En América existen alrededor de 5,3 millones de hectáreas de producción de arroz, la consecuencia del cambio climático y las reducciones de agua para este cultivo se ve en términos de implementar técnicas que garanticen la producción (Fontagro, 2019).

En Latinoamérica y la producción de arroz se da en 23 países y según la FAO 2014 fue de 25.2 millones de toneladas (el 3,3\% de la producción mundial), del cual Brasil fue el primero con un total de 12.17 millones de toneladas con 2.3 millones de hectáreas. Perú tiene el segundo lugar con 2.89 millones de toneladas.

\subsection{QUE ES EL SISTEMA INTENSIVO DE LA PRODUCCIÓN DE ARROZ}

El Sistema Intensivo de la Producción de Arroz es una metodología que pretende intensificar la producción de arroz, con la disminución del riego y el uso de químicos, y sacarle un mayor rendimiento al cultivo que es muy consumido a nivel mundial. En 1983 los elementos principales fueron reunidos por el padre Jesuita Francés Henri de Laulanié en la isla de Madagascar, después de 20 años y experimentación con arroz, pero su implementación se dio si no hasta unos 10 a 20 años después de sus estudios (Mendoza, 2017).

El Sistema Intensivo de la Producción de Arroz es una metodología que busca incrementar la productividad del arroz, mediante la eficiencia del manejo de las plántulas, reduce la necesidad en termino de semillas, agua y en otro caso la mano de obra, este sistema reduce la competición entre plantas y genera 
un mayor beneficio al grano al obtener más nutrientes del suelo, un mejor control de la maleza y resistencia a plagas, enfermedades y a los cambios climáticos (Uphoff, 2015).

Existen dos principios básicos que son las plantas jóvenes y la oxigenación de las raíces, lo cual produce plantas más robustas de arroz con panículas pesadas. El trasplante de las plántulas es dentro de los 10 días de edad, de esta manera se está ejecutando antes de la emergencia del primer macollo que crece en el tallo primario, las plántulas tienen tiempo necesario en el campo para que de esta manera la formación del tallo comience hacer óptimo, de esta forma los primeros macollos estén en sus mejores condiciones y den origen a su potencial macollamiento (Shiguango, 2014).

\subsection{PAÍSES DONDE SE IMPLEMENTA ESTE SISTEMA}

Debido que en Latinoamérica son tierras de buena calidad y teniendo climas que favorecen a la producción agrícola, la implementación del sistema intensivo de la producción de arroz es muy buena, países como Colombia, República Dominicana, Panamá, Nicaragua, Costa Rica, Cuba, Trinidad y Tobago, Ecuador, Venezuela y Argentina, estén implantando esta técnica de producción que busca es maximizar ganancias.

El implemento de este sistema ha generado beneficios tanto social y económico, por el uso más eficaz de agua y suelo, una mayor productividad con menos insumos y más resistentes a diferentes acontecimientos. Esto genera una mayor demanda de personal para el trasplante hacia las piscinas y el control de maleza. (IICA, 2017).

\subsection{BENEFICIOS DEL SISTEMA INTENSIVO DE LA PRODUCCIÓN ARROZ.}

Dentro de los beneficios que tiene el sistema intensivo de la producción de arroz, son muy variables y depende de las exigencias del productor al aplicar los principios fundamentales de este sistema de los cuales (Mendoza, 2017). Menciona las principales ventajas de sistema intensivo de la producción de arroz:

1. El ahorro considerable en el uso de semilla, $80 \%$ de reducción (se usan de 10 a 12 kg/ha).

2. Ahorro en el uso de agua de riego (40-60\%), lo cual garantiza la conservación en tiempos de sequias.

3. Las plantas generan raíces y tallos más fuertes.

4. Mayor macollamiento por planta.

5. Se reduce el ciclo de cultivo.

6. Menor ataque de plagas.

7. Uso de semilleros.

8. Cero o poco volcamiento del arroz. 
9. Reducción del gas metano, debido al no tener inundado el terreno durante el ciclo de producción.

10. Un ambiente más sano por la reducción del uso de agroquímico.

11. Excelente calidad del grano.

12. Promueve la conservación del carbono en el suelo.

13. Reduce los costos de producción y obtener mayor utilidad.

\subsection{EL ARROZ EN EL ECUADOR}

En Ecuador la producción de arroz representa una importancia socioeconómica según el INEC, se cultivan alrededor de 411459 hectáreas, la gran parte proviene de pequeños productores los mismos que su producción es prácticamente artesanal, a comparación de diferentes países donde la implementación del Sistema Intensivo de la Producción de Arroz ya está dando beneficios.

La figura 1 indica el porcentaje de donde esta localizadas la cantidad de microempresas productoras de arroz en Ecuador en 2020, siendo la provincia del Guayas con: (77\%), Los Ríos (14\%), Manabí (4\%) y otros (5\%) (CFN, 2021).

Figura 1 Porcentajes de Microempresas de arroz en Ecuador 2020

Microempresas

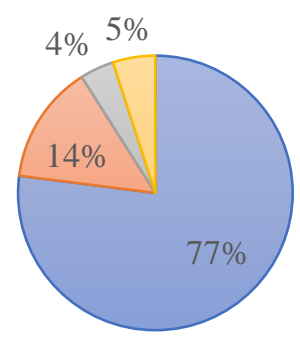

口Guayas. $\quad \square$ Los Ríos. $\square$ Manabí. $\square$ Otros.

\subsection{SISTEMA TRADICIONAL DE PRODUCCIÓN DE ARROZ EN ECUADOR}

Dentro de la producción tradicional de arroz se encuentran distintos métodos de cultivos, los mismos que generan diferentes cantidades según su implementación, dentro de tecnificado, por lo general es puesto en prácticas por grandes productores, los mismos que llegan hasta las $7 \mathrm{Tm} / \mathrm{ha}$. Los semitecnificadas, producen un promedio de 3 a $4 \mathrm{Tm} / \mathrm{ha}$. Y las no tecnificadas llegan a tener de 3 y menos de 3 Tm/ha, (Rodriguez, 2010).

Según la entrevista realizada por el Banco Central del Ecuador, en el 2019 en las provincias de Manabí, Loja, Los Ríos, El Oro y Cañar, 64\% de los productores señalaron que la producción fue mayor que en años anteriores, en cambio el $24 \%$ señaló que se mantienen igual al año anterior y el $7 \%$ manifestó 
que su producción fue menor. Mientras los rendimientos por hectárea fueron mayores para los informales con un $57 \%$, el $34 \%$ señalo que se mantuvo y el $9 \%$ que fueron menores que los años anteriores. El volumen de producción fue mayor para $68 \%$ de los productores mientras que el $32 \%$ mantuvo que fue igual (BCE, 2020).

\subsection{COSTOS DE PRODUCCIÓN DEL SISTEMA TRADICIONAL DE PRODUCCIÓN DE ARROZ}

Según el diario el Comercio en 2021 la inversión por hectárea de sembrío de arroz es de \$1 100 a \$1300 esto manifiestan los productores de cantones de Guayas, lo cual les permite una ganancia liquida de $\$ 380$ por cada periodo de 3 o 4 meses que dura el ciclo, debido a los diferentes factores que inciden en comercialización y preproducción en el cual la parte arrocera de Ecuador es afectada con los bajos precios que se paga, a lo contrario de los elevados costos de producción (Castellanos, 2021).

\section{MATERIALES Y MÉTODOS}

Mediante la revisión bibliográfica y documental, la misma que se dio por la revisión de artículos relacionados al tema de investigación, los cuales fueron tomado de algunas fuentes científicas y páginas que garantizan la veracidad de la investigación, dentro de los materiales utilizados están tablas creadas en Excel, Word y datos de diferentes fuentes como FAO, FAOSTAT, FONTAGRO y diario El Universo, el uso del recurso Internet que hace más eficiente la recaudación de información.

La investigación es de carácter descriptivo, en base de los datos de las diferentes fuentes relacionadas con el tema, teniendo como búsqueda en sitios como Scopus, Scielo, Google Académico y plataforma del IICA.

\section{RESULTADOS Y DISCUSIÓN}

La falta de recursos económicos en la producción de arroz en Ecuador

Una de las necesidad que tiene Ecuador es la fuente de alimentación y siendo el arroz uno de los cereales más consumido, esto genera que los productores de esta gramínea busquen implementar nuevas técnicas que mejoren la productividad de las plantaciones de arroz, debido que representa en gran parte de la alimentación familiar convirtiéndose en un producto vital dentro de los hogares, en Ecuador gran parte de esta producción es dada por pequeños productores y su comercialización es en su mayoría local.

La implementación del sistema intensivo de la producción de arroz es un novedoso sistema de producción, que busca maximizar los resultados en la producción, utilizando menos recursos para la implementación del cultivo, en Latinoamérica países como: Ecuador, Colombia, Perú, Venezuela ven resultados favorables en el sistema de la producción de arroz a comparación del sistema tradicional. 
La tabla 1 se especifica que Ecuador durante el año 2020 registro un total de 77 empresas conciliadas legal mente como productoras de arroz, dentro de estas se observa que la Microempresa es la de mayor alcance con 46 empresas registradas, dentro de la pequeña empresa se tiene 20 registradas, la Mediana Empresa con 7, Grande solo 3 y no Definida 1, debido que la producción de arroz genera márgenes de utilidad bajos, cada año son menos las empresas que continúan cultivando arroz y cambian sus cultivos por otros de menor costos de producción.

Tabla 1. Número de empresas que cultivan arroz en Ecuador en el 2020

\begin{tabular}{|c|c|}
\hline Tamaño de la Empresa & Número de Empresas 2020 \\
\hline Grande & 3 \\
\hline Mediana & 7 \\
\hline Pequeño & 20 \\
\hline Microempresa & 46 \\
\hline No Definido & 1 \\
\hline Total & 77 \\
\hline
\end{tabular}

Fuente: CFN (2021).

La tabla 2 demuestra que las toneladas de producción dentro del 2017 al 2020 en sudamericana han crecido, en el 2017 la producción de arroz alcanzo 2.719.075 toneladas, dentro del 2018 y 2019 la producción prácticamente se mantiene, pero en el 2020 su producción alcanza las 4053003 toneladas, incrementando a casi el doble de la producción del 2017.

Tabla 2. Producción en toneladas en Sudamérica en el periodo 2017 - 2020

\begin{tabular}{|c|c|}
\hline \multicolumn{2}{|c|}{ Producción de Arroz en Sudamérica } \\
\hline Años & Toneladas \\
\hline 2017 & 2719075 \\
\hline 2018 & 3251384 \\
\hline 2019 & 3318704 \\
\hline 2020 & 4053003 \\
\hline
\end{tabular}

Fuente: FAOSTAT.

La tabla 3 demuestra que inversión por hectárea para el cultivo de arroz en Ecuador es alta, esto genera que muchos productores cambien su cultivo por otros más rentables para obtener una mayor ganancia. Se puede notar que el costo de producción por hectárea de arroz ha disminuido, esto no garantiza el éxito de la producción arroz, debido a diferentes factores externos que inciden en el cultivo en unos como son las plagas que generan millos de perdidas al no ser controladas rigurosamente y los cambios bruscos de clima que generan perdidas del producto y económicas en los productores. 
Tabla 3. Inversión de la producción tradicional de arroz en Ecuador

\begin{tabular}{|c|c|c|}
\hline \multicolumn{3}{|c|}{ Inversión de la producción tradicional de arroz en Ecuador } \\
\hline Años & Inversión por Hectáreas $\$$ & Ganancia por Hectárea $\$$ \\
\hline 2017 & $\$ 1.800$ & $\$ 395$ \\
\hline 2018 & $\$ 1.800$ & $\$ 292$ \\
\hline 2019 & $\$ 1.200$ & $\$ 321$ \\
\hline 2020 & $\$ 1.200$ & $\$ 359$ \\
\hline 2021 & $\$ 1.100$ & $\$ 380$ \\
\hline
\end{tabular}

Fuente: El Universo.

La tabla 4 de FONTAGRO, indica las ventajas que tiene este nuevo sistema a comparación del tradicional, generando una mayor garantía a los productores que ven como una solución de reducir los costos al momento de producir arroz. Con un menor tiempo de trasplante garantizando una mayor producción de macollos y tallos fuertes, menor dependencia de agroquímicos lo cual genera un cultivo saludable, mejor control de la maleza dentro de la distancia entre planta la misma que reduce la competencia de nutriente entre plantas al tener un sistema de 30x30 o 25x25 (distancia entre planta), mayor cantidad de raíces (más largas, profundas y sanas).

Tabla 4. diferencia entre el Sistema Tradicional y el Sistema Intensivo de la Producción de Arroz

\begin{tabular}{|c|c|c|}
\hline \multicolumn{2}{|c|}{ Diferencia entre el Sistema Tradicional y el Sistema Intensivo de la Producción de Arroz } \\
\hline Labores Agrícolas & Tradicional & Sistema Intensivo de la Producción de Arroz \\
\hline Preparación del terreno & Igual & Igual \\
\hline Días a trasplante & 20 días (4 Hojas) & 10 días (2 hojas) \\
\hline Control de maleza & Químico & Mecánico \\
\hline Manejo de riego & Inundación & Orgánica \\
\hline Fertilización & Química & Ninguno \\
\hline Control de enfermedades & Químico & Ninguno \\
\hline Control de insectos & Químico & 110 días \\
\hline Días a cosechar & 110 días & Fuente: FONTAGRO. \\
\hline \multicolumn{3}{|c|}{} \\
\hline
\end{tabular}

La tabla 5 demuestra 6 tratamientos donde los impares están representados por el sistema tradicional y los pares están con el sistema intensivo de la producción de arroz, se nota que el rendimiento de quintal por hectárea es superior en el sistema intensivo, su precio por quinal también es superior al tradicional, lo cual garantiza un beneficio neto mayor que el sistema tradicional.

Tabla 5. Comparación entre el sistema tradicional y sistema intensivo de la producción de arroz Tratamientos entre sistema tradicional y el sistema intensivo de producción de arroz

\begin{tabular}{|c|c|c|c|c|c|c|}
\hline & \multicolumn{7}{|c|}{ Tratamientos } \\
\hline & T1 & T2 & T3 & T4 & T5 & T6 \\
\hline Rend. qq/ha & 115 & 121 & 116 & 123 & 116 & 124 \\
\hline Rend. Ajust. 10\% & 103.5 & 108.9 & 104.4 & 110.7 & 104.4 & 111.6 \\
\hline Precio qq/ USD 28.00 & 2898 & 3049.2 & 2923.2 & 3099.6 & 2923.2 & 3124.8 \\
\hline Costo Variable & \multicolumn{7}{|c|}{} \\
\hline Cultivo de arroz (Saca 120 libra.) & 20.5 & 20.5 & 23 & 23 & 22.5 & 22.5 \\
\hline Sistema de siembra (jornal) & 120 & 96 & 120 & 96 & 120 & 96 \\
\hline Total, de Costos Variables & 140.5 & 116.5 & 143 & 119 & 142.5 & 118.5 \\
\hline Beneficio Neto & 2757.5 & 2932.7 & 2780.6 & 2980.6 & 2780.7 & 3006.3 \\
\hline
\end{tabular}

Fuente: Macías López Javier Oswaldo (2015). 
Dentro de la Tabla 6, se observa que la producción de arroz en el 2017 alcanzó 499.9 millones de toneladas y a consideración del 2021 que alcanzó 518.4 millones toneladas, obteniendo un crecimiento favorable lo cual contribuye que la comercialización aumenta, en 2017 se nota que ha crecido de un 48.7 y dentro del 2021 las exportaciones fueron dadas 51.4 millones de toneladas. Dentro de los principales exportadores de este producto se encuentran un crecimiento porcentual dentro del 2017 los 5 países contribuyentes de este producto son India, Pakistán, Tailandia, Estados Unidos, Vietnam, con 18.1\% de contribución y dentro del 2021 alcanzaron el $26.8 \%$ de la producción mundial.

Tabla 6. Mercado Mundial de Arroz

\begin{tabular}{|c|c|c|c|c|c|c|}
\hline \multicolumn{7}{|c|}{ Mercado Mundial de Arroz } \\
\hline & \multirow{3}{*}{$2017 / 2018$} & \multirow{3}{*}{$2018 / 2019$} & \multirow{3}{*}{$2019 / 2020$} & \multirow{3}{*}{$2020 / 2021$} & \multicolumn{2}{|c|}{$2021 / 2022$} \\
\hline & & & & & Anterior & Actual \\
\hline & & & & & $\begin{array}{c}04 \text { nov. } \\
2021\end{array}$ & $\begin{array}{c}02 \text { dic. } \\
2021\end{array}$ \\
\hline \multicolumn{7}{|c|}{ Millones de Toneladas } \\
\hline Producción 1/ & 499.9 & 508.2 & 502.9 & 513.7 & 518.2 & 518.4 \\
\hline Suministros 2/ & 673.6 & 685.1 & 689.1 & 699.5 & 705.3 & 705.4 \\
\hline Utilización & 498.0 & 501.3 & 501.8 & 510.7 & 518.8 & 518.9 \\
\hline Comercio 3/ & 48.7 & 44.2 & 45.6 & 49.0 & 51.3 & 51.4 \\
\hline Existencias al final del ejercicio 4/ & 176.9 & 186.2 & 185.8 & 187.0 & 187.6 & 187.7 \\
\hline \multicolumn{7}{|c|}{ Por ciento } \\
\hline Relación mundial existencias utilizadas & 35.3 & 37.1 & 36.4 & 36.0 & 35.8 & 35.8 \\
\hline $\begin{array}{l}\text { Relación existencias - desaparición de los } \\
\text { principales exportadores 5/ }\end{array}$ & 18.1 & 22.5 & 25.8 & 26.9 & 26.7 & 26.8 \\
\hline
\end{tabular}

Fuente: FAO (2021).

Los diferentes estudios realizados por medio del Instituto Internacional de Cooperación para la Agricultura (IICA), demuestran que la implementación del sistema intensivo de producción de arroz, garantiza el crecimiento de la producción con menor inversión, obteniendo una mayor rentabilidad que favorece a los productores que se ven limitados y en muchos casos cambian su cultivo por lo difícil que cuesta mantener esta producción donde el índice de riesgos es alto.

\section{CONCLUSIONES}

La producción de arroz en Ecuador es prácticamente tradicional este sistema genera pérdidas en el cultivo debido a lo sensible que es el arroz, lo contrario del sistema intensivo de la producción de arroz, que genera incrementos de la producción, reduce el costo de inversión por lo cual garantizan un mayor ingreso. La implementación del sistema intensivo de la producción de arroz en Ecuador no es muy conocida por los productores, los datos obtenidos dentro de la investigación demuestran que este nuevo sistema es de gran importancia por su alto grado de confiabilidad que han obtenido los diferentes países que han implementado este sistema de producción. 
Debido a los incrementos de inversión del sistema tradicional es necesario implementar el sistema intensivo de la producción de arroz en Ecuador porque genera mayores rendimientos en la cosecha del producto, una mayor eficiencia de los recursos, amigable con el medio ambiente, y mayores ganancias para el productor. 


\section{REFERENCIAS}

BCE. (Abril de 2020). REPORTE DE COYUNTURA SECTORIAL AGROPECUARIO. 48. Recuperado el 01 de $\quad$ Enero de $2022, \quad$ de https://contenido.bce.fin.ec/documentos/PublicacionesNotas/Catalogo/Encuestas/Coyuntura/Integradas/ etc201904.pdf

Bernis, J. M., \& Pàmies, C. B. (2004). VARIEDADES Y MEJORA DEL ARROZ (Oryza sativa, L.) (Universidad Internacional de Cataluña ed.). UIC - Campus de l'Ebre. Obtenido de http://espacio.uned.es/fez/eserv/bibliuned:UNEDCentroAsociadoTortosa-Libros-

5025/Franquet_Bernis_JoseMaria_Variedades.pdf

Castellanos, F. (19 de Julio de 2021). El Universo. Recuperado el 04 de 01 de 2022, de https://www.eluniverso.com: https://www.eluniverso.com/noticias/ecuador/con-un-precio-de-28-porsaca-de-210-libras-de-arroz-en-cascara-productores-reciben-una-ganancia-de-380-por-hectarea-en-treso-cuatro-meses-nota/

CFN. (Julio de 2021). /www.cfn.fin.ec. Recuperado el 12 de Enero de 2022, de https://www.cfn.fin.ec/wpcontent/uploads/downloads/biblioteca/2021/fichas-sectoriales-3-trimestre/Ficha-Sectorial-Arroz.pdf

Chang, J. V. (2008). CULTIVO DE ARROZ SISTEMA INTENSIFICADO SICA-SRI EN ECUADOR. ADMICORPORACION, 5.

Fontagro. (2019). fontagro. Recuperado el 07 de 01 de 2022, de Fontagro: https://www.fontagro.org/new/proyectos/arrozmasproductivo/es

IICA. (10 de Octubre de 2017). Proyecto Cultivar más con menos: Adaptación, validación y promoción del Sistema Intensivo del Cultivo Arrocero (SRI) en las Américas como una respuesta al cambio climático. 5. Recuperado el $06 \quad 01 \quad$ de $\quad 2022, \quad$ de http://sri.ciifad.cornell.edu/spanish/Taller_Regional_SRI_101017.pdf

Mendoza, D. A. (2017). Guía para el establecimiento y monitoreo del cultivo de arros bajo la metodologia SRI. FONTAGRO, 7. Recuperado el 05 de 01 de 2021

Quijije, B. A., Carvajal, S. J., Garcia, K. E., \& Cedeño, W. B. (04 de 03 de 2019). Costo, volumen y utilidad del cultivo de arroz, cantón Samborondón (Ecuador). Espacios, 40(7), 10. Obtenido de http://www.revistaespacios.com/a19v40n07/a19v40n07p16.pdf

Rodriguez, R. L. (2010). SITUACION ARROCERA ECUATORIANA. CORPORACIÓN DE INDUSTRIAS ARROCEROS DEL ECUADOR, 4. Recuperado el 04 de 02 de 2022, de http://www.acpaarrozcorrientes.org.ar/Jornadas-2010/11.pdf

Shiguango, M. F. (25 de Agosto de 2014). COMPARACIÓN AGRONÓMICA DE OCHO CULTIVARES DE ARROZ EN DOS TIPOS DE SUELO BAJO EL MÉTODO SRI. (F. Eison Valdiviezo, Ed.) 14. Recuperado el 05 de 01 de 2022

Uphoff, N. (22 de Julio de 2015). SISTEMA DE INTENSIFICACIÓN DEL CULTIVO DEL ARROZ (SRI). IICA, 3. Recuperado el 06 de 01 de 2022, de http://sri.ciifad.cornell.edu/spanish/IICA_SRI_Preguntas_Frecuentesz_Uphoff042916.pdf 